\title{
A clip in the right place: successful endoscopic submucosal dissection of a cecal tumor exhibiting the muscle-retracting sign
}

Among the challenges encountered during endoscopic submucosal dissection (ESD), firm "retraction" of the muscularis propria towards the tumor (the "muscleretracting" sign) can lead to non-curative resection, failure to complete ESD, or perforation [1]. Peranal endoscopic myectomy has been introduced as a means for dealing with such lesions, its merit however is currently limited to the lower rectum, where the muscularis propria is thicker compared with the rest of the colon [2]. Based on the above, we decided to illustrate a technical variation of ESD that was used to achieve an $\mathrm{R} 0$ resection

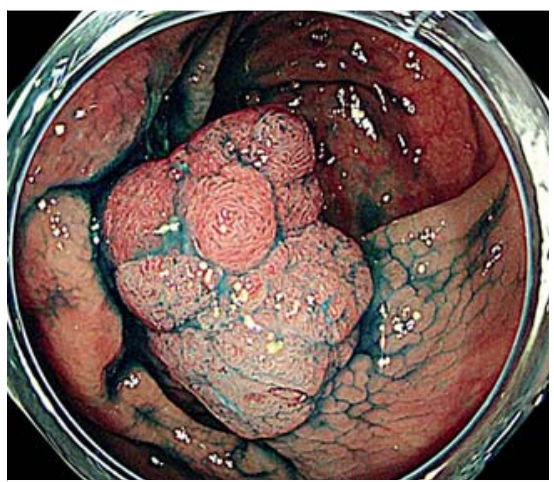

- Fig. 1 Endoscopic view showing a protruded-type tumor in the cecum. for a cecal type 0 -Is tumor with the muscle-retracting sign ( $>$ Fig. 1 ; $\triangleright$ Video 1 ).

During colonoscopy, an $18-\mathrm{mm} 0$-Is tumor was identified in the cecum of an 80 -year-old man with a history of poststroke paralysis. Because of the patient's age and underlying disease, ESD was performed. During ESD, a muscle-retracting area was recognized in the center of the lesion and the surrounding submucosa was dissected to expose this area. The mucosal incision was then completed, leaving only the muscle-retracting area temporarily intact ( $\mathbf{F i g .} \mathbf{2}$ a). In order to achieve R0 resection and prevent perforation, a reopenable hemoclip was anchored onto the muscle-retracting area as close as possible to the muscularis propria ( $>$ Fig. $\mathbf{2}$ b). The remaining tissue above the clip was then dissected, while avoiding contact between the ESD knife and the metal "arms" of the hemoclip
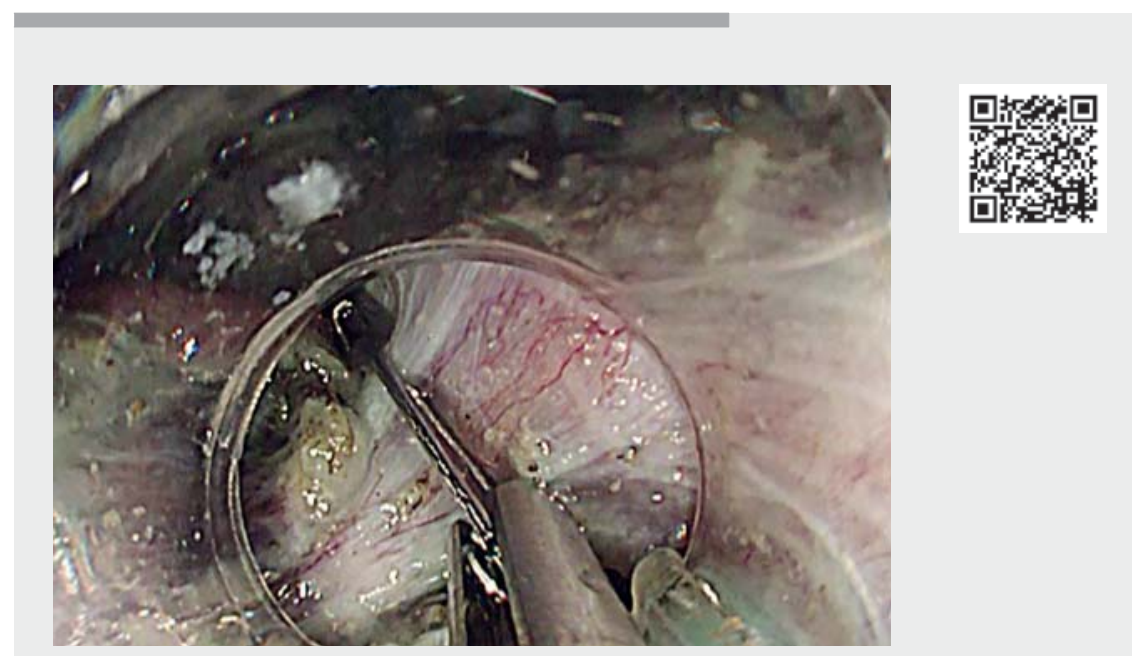

Video 1 Endoscopic submucosal dissection of a cecal protruded-type tumor with the muscle-retracting sign.
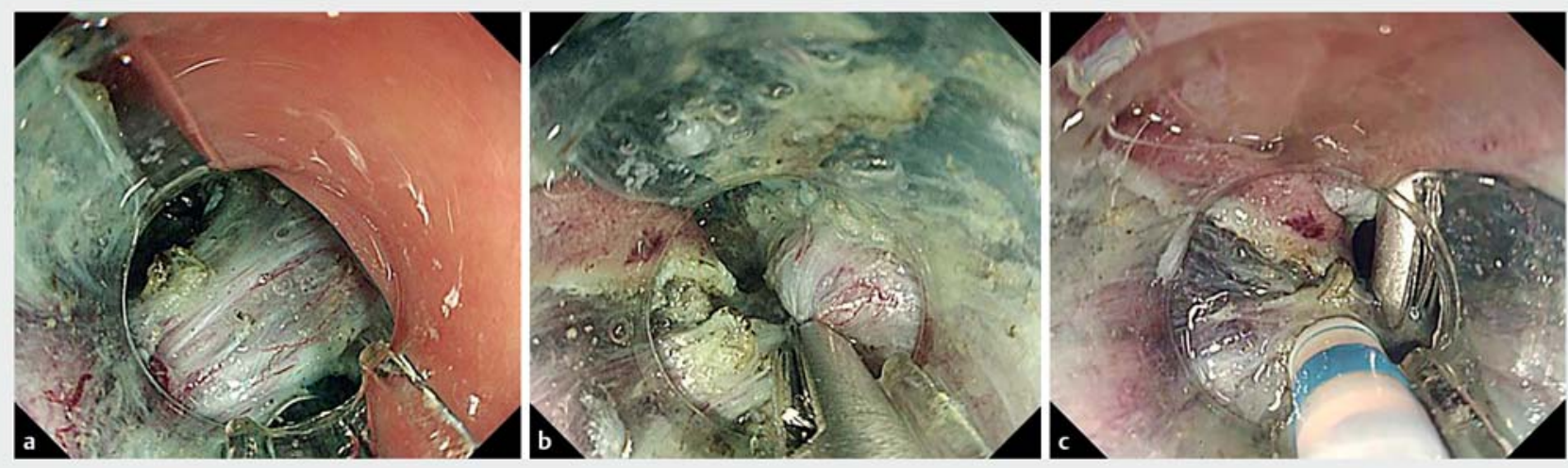

Fig. 2 Endoscopic images during the endoscopic submucosal dissection procedure showing: a exposure of the muscle-retracting area; b clipping of the muscle-retracting area with a reopenable hemoclip; c dissection above the hemoclip. 


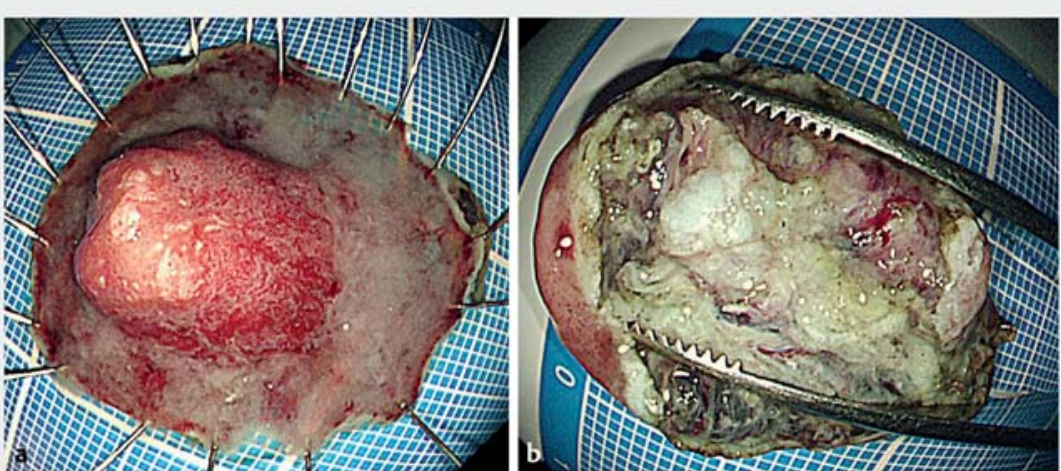

Fig. 3 Macroscopic appearance of the resected specimen showing: a the mucosal surface; b the resected surface.

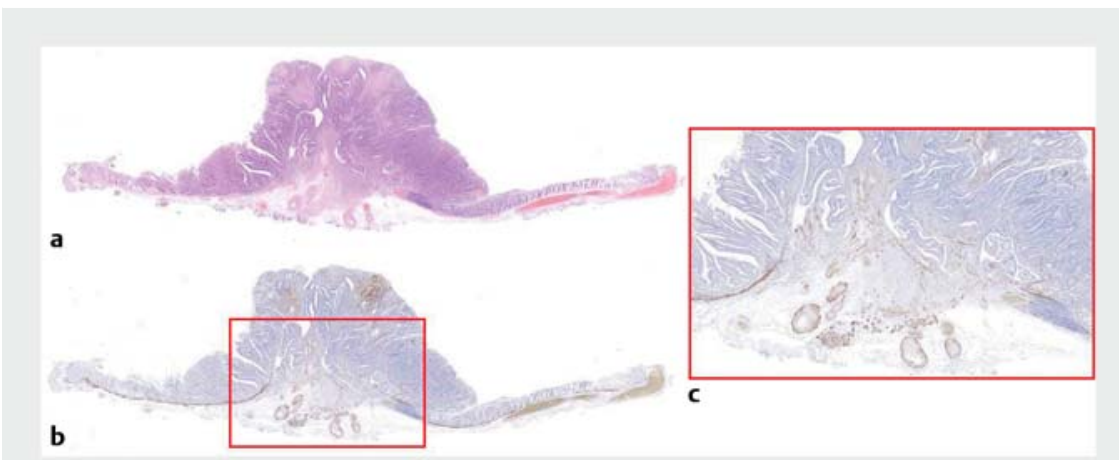

- Fig. 4 Histopathological appearance of a section of the deepest region: a with hematoxylin and eosin (H\&E) staining; $\boldsymbol{b}$ with desmin staining; $\mathbf{c}$ at higher magnification, showing deep submucosal invasion just above the muscularis propria, which was consistent with a type 0 -Is, $15 \times 15-m m$ adenocarcinoma (tub1, pT1b [4500 $\mu \mathrm{m}]$, ly0, v0, BD1, pHM0, pVM0).

( $\triangleright$ Fig. 2c). ESD was completed without perforation, and the ESD defect was completely closed with hemoclips to prevent delayed perforation.

The muscle-retracting area could be identified on the resected surface of the specimen ( Fig.3). Histopathologic examination revealed tumor invasion into the submucosa $(4500 \mu \mathrm{m})$ just above the muscularis propria (pT1b), but the resection margins were negative and no lymphovascular invasion was documented (-Fig.4).

In conclusion, upon identification of the muscle-retracting sign during ESD, clipping at the base of the muscle-retracting area and dissection above the clip can prevent perforation while maximizing resection depth to ensure an $\mathrm{R} 0$ resection.

Endoscopy_UCTN_Code_TTT_1AQ_2AD

\section{Competing interests}

The authors declare that they have no conflict of interest.

The authors

Hisashi Fukuda', Yuka Kowazaki ${ }^{1}$, Itaru Saito', Shinichi Hirooka ${ }^{2}$, Tomohiro Kurokawa ${ }^{3}$,

Norio Kanzaki ${ }^{3}$, Anastasios C. Manolakis ${ }^{4,5}$

1 Department of Gastroenterology, Jyoban Hospital, Tokiwa Foundation, Fukushima, Japan

2 Department of Pathology, Jyoban Hospital, Tokiwa Foundation, Fukushima, Japan

3 Department of Surgery, Jyoban Hospital, Tokiwa Foundation, Fukushima, Japan

4 University of Thessaly, School of Medicine, Larissa, Greece

5 Department of Gastroenterology, University Hospital of Larissa, Larissa, Greece
Corresponding author

Hisashi Fukuda, MD

Department of Gastroenterology, Jyoban Hospital, Tokiwa Foundation, 57 Jyoban kamiyunagayamachi kaminodai, Iwaki, Fukushima, 972-8322, Japan

r0849hf@jichi.ac.jp

References

[1] Toyonaga T, Tanaka S, Man-I M et al. Clinical significance of the muscle-retracting sign during colorectal endoscopic submucosal dissection. Endosc Int Open 2015; 3: E246E251

[2] Toyonaga T, Ohara Y, Baba S et al. Peranal endoscopic myectomy (PAEM) for rectal lesions with severe fibrosis and exhibiting the muscle-retracting sign. Endoscopy 2018; 50: 813-817

Bibliography

Endoscopy 2022; 54: E520-E521

DoI $10.1055 / \mathrm{a}-1656-9340$

ISSN 0013-726X

published online 25.10.2021

(C) 2021. Thieme. All rights reserved.

Georg Thieme Verlag KG, Rüdigerstraße 14, 70469 Stuttgart, Germany

\section{ENDOSCOPY E-VIDEOS}

https://eref.thieme.de/e-videos

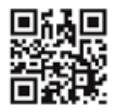

Endoscopy E-Videos is an open access online section, reporting on interesting cases and new techniques in gastroenterological endoscopy. All papers include a high quality video and all contributions are freely accessible online. Processing charges apply (currently EUR 375), discounts and wavers acc. to HINARI are available.

This section has its own submission website at https://mc.manuscriptcentral.com/e-videos 